\title{
Invariant Solutions and Conservation Laws of the $(2+1)$-Dimensional Boussinesq Equation
}

\author{
Wenjuan Rui, ${ }^{1}$ Peiyi Zhao, ${ }^{2}$ and Yufeng Zhang ${ }^{1}$ \\ ${ }^{1}$ College of Science, China University of Mining and Technology, Xuzhou 221116, China \\ ${ }^{2}$ Shandong Provincial Academy of Education Recruitment and Examination, Jinan 250011, China \\ Correspondence should be addressed to Wenjuan Rui; ruiwj@126.com
}

Received 8 June 2014; Accepted 17 July 2014; Published 7 August 2014

Academic Editor: Huanhe Dong

Copyright (c) 2014 Wenjuan Rui et al. This is an open access article distributed under the Creative Commons Attribution License, which permits unrestricted use, distribution, and reproduction in any medium, provided the original work is properly cited.

\begin{abstract}
Invariant solutions and conservation laws of the $(2+1)$-dimensional Boussinesq equation are studied. The Lie symmetry approach is used to obtain the invariant solutions. Conservation laws for the underlying equation are derived by utilizing the new conservation theorem and the partial Lagrange approach.
\end{abstract}

\section{Introduction}

In recent years, searching for explicit solutions of nonlinear evolution equations (NEEs) has attracted the attention of many mathematicians and physicists. Particularly, various effective methods have been used to explore different kinds of solutions of NEEs, such as the inverse scattering method [1], the Darboux transformation [2] and the Bäcklund transformation [3], the Hirota method [4], the homogeneous balance method $[5,6]$, the similarity reduced method $[7$, 8], the tanh method [9], and the sine-cosine method [10]. But up to now a unified method that can be used to deal with all types of NEEs has not been discovered. Among the above mentioned methods, the Lie symmetry method is one of the most effective methods to determine solutions of differential equations. In the past decades, there have been considerable developments in symmetry methods for differential equations [11, 12].

In the present paper we consider the $(2+1)$-dimensional Boussinesq equation:

$$
u_{t t}-u_{x x}-u_{y y}-\left(u^{2}\right)_{x x}-u_{x x x x}=0 .
$$

A well-known soliton equation is the Boussinesq equation:

$$
u_{t t}-u_{x x}-3\left(u^{2}\right)_{x x}-u_{x x x x}=0 .
$$

Equation (2) was introduced by Boussinesq to describe motions of long waves in shallow water $[13,14]$. It also appears in a wide variety of physical systems such as nonlinear lattice waves, iron sound waves in plasma, and vibrations in a nonlinear string. For the transonic speed perturbations, by neglecting the interaction of waves moving in the opposite directions, the Boussinesq equation (2) can be reduced to the KdV equation. Equation (2) itself is also a dimensional reduction of the KP equation in the moving frame. Moreover, the Boussinesq equation (2) is completely integrable and admits inverse scattering. Owing to its profound importance and nice mathematical properties, a great deal of research work has been invested in recent years for the study of the Boussinesq equation. Krishnan et al. [15] studied the dynamics of shallow water waves that are governed by the Boussinesq equations. Yang et al. [16] obtained solutions of homogeneous and inhomogeneous dissipative Boussinesq equation by using the modified Jacobi elliptic function expansion method and the pseudospectral method. However, there has also been a growing interest in the study of NEEs in multidimensions, especially in $(1+2)$ and $(1+3)$ dimensions. To find some exact soliton solutions in higher dimensions is much more difficult than in $1+1$ dimensions. Recently, El-Sayed and Kaya [17] used the decomposition method to obtain the exact solitary-wave solutions of (1). Senthilvelan [18] obtained the travelling wave solutions for $(2+1)$ dimensional Boussinesq equation and $(3+1)$-dimensional KP equation by homogeneous balance method and explored certain new solutions of the equations. Chen et al. [19] 
obtained many explicit exact solutions of (1) by using the new generalized transformation in homogeneous balance method. More new double periodic and multiple soliton solutions are obtained for the generalized $(2+1)$-dimensional Boussinesq equation [20].

The main goal of this paper is to use the Lie symmetry method $[21,22]$ to obtain the invariant solutions. In addition to this, conservation laws will be derived for (1) by using the new conservation theorem $[23,24]$ and the partial Lagrange approach $[25,26]$.

The outline of this paper is as follows. In Section 2, we present symmetry group analysis and group-invariant solutions of (1). In Section 3, the conservation laws for (1) are established. Finally, some conclusions are given in Section 4.

\section{Method of Lie Symmetries}

2.1. Preliminaries. In this section we briefly present the notation and pertinent results used in this paper $[21,22]$.

Consider a $k$ th-order system of PDEs of $n$ independent variables $x=\left(x^{1}, x^{2}, \ldots, x^{n}\right)$ and $m$ dependent variables $u=$ $\left(u^{1}, u^{2}, \ldots, u^{m}\right)$ :

$$
E_{\alpha}\left(x, u, u_{(1)}, \ldots, u_{(k)}\right)=0, \quad \alpha=1, \ldots, m,
$$

where $u_{(1)}, u_{(2)}, \ldots, u_{(k)}$ denote the collections of all first, second,..., $k$ th-order partial derivatives; that is, $u_{i}^{\alpha}=$ $D_{i}\left(u^{\alpha}\right), u_{i j}^{\alpha}=D_{j} D_{i}\left(u^{\alpha}\right), \ldots$, respectively, with the total derivative operator with respect to $x^{i}$ given by

$$
D_{i}=\frac{\partial}{\partial x^{i}}+u_{i}^{\alpha} \frac{\partial}{\partial u^{\alpha}}+u_{i j}^{\alpha} \frac{\partial}{\partial u_{j}^{\alpha}}+\cdots, \quad i=1, \ldots, n,
$$

where the summation convention is used whenever appropriate.

The Euler-Lagrange operator, for each $\alpha$, is given by

$$
\frac{\delta}{\delta u^{\alpha}}=\frac{\partial}{\partial u^{\alpha}}+\sum_{s \geq 1}(-1)^{s} D_{i_{1}} \cdots D_{i_{s}} \frac{\partial}{\partial u_{i_{1} i_{2} \cdots i_{s}}^{\alpha}},
$$

and the Lie-Bäcklund operator is

$$
X=\xi^{i} \frac{\partial}{\partial x^{i}}+\eta^{\alpha} \frac{\partial}{\partial u^{\alpha}}, \quad \xi^{i}, \eta^{\alpha} \in \mathscr{A}
$$

where $\mathscr{A}$ is the space of differential functions. The operator (6) is an abbreviated form of infinite formal sum:

$$
X=\xi^{i} \frac{\partial}{\partial x^{i}}+\eta^{\alpha} \frac{\partial}{\partial u^{\alpha}}+\sum_{s \geq 1} \zeta_{i_{1} i_{2} \cdots i_{s}}^{\alpha} \frac{\partial}{\partial u_{i_{1} i_{2} \cdots i_{s}}^{\alpha}},
$$

where the additional coefficients are determined uniquely by the prolongation formulae:

$$
\begin{gathered}
\zeta_{i}^{\alpha}=D_{i}\left(W^{\alpha}\right)+\xi^{j} u_{i j}^{\alpha}, \\
\zeta_{i_{1} \cdots i_{s}}^{\alpha}=D_{i_{1}} \cdots D_{i_{s}}\left(W^{\alpha}\right)+\xi^{j} u_{j i_{1} \cdots j_{s}}^{\alpha}, \quad s>1,
\end{gathered}
$$

in which $W^{\alpha}$ is the Lie characteristic function:

$$
W^{\alpha}=\eta^{\alpha}-\xi^{j} u_{j}^{\alpha}
$$

One can write the Lie-Bäcklund operator (7) in characteristic form as follows:

$$
X=\xi^{i} D_{i}+W^{\alpha} \frac{\partial}{\partial u^{\alpha}}+\sum_{s \geq 1} D_{i_{1} i_{2} \cdots i_{s}}\left(W^{\alpha}\right) \frac{\partial}{\partial u_{i_{1} i_{2} \cdots i_{s}}^{\alpha}} .
$$

The Noether operators associated with a Lie-Bäcklund symmetry operator $X$ are given by

$$
\begin{array}{r}
N^{i}=\xi^{i}+W^{\alpha} \frac{\delta}{\delta u_{i}^{\alpha}}+\sum_{s \geq 1} D_{i_{1} i_{2} \cdots i_{s}}\left(W^{\alpha}\right) \frac{\delta}{\delta u_{i i_{1} i_{2} \cdots i_{s}}^{\alpha}}, \\
i=1,2, \ldots, n,
\end{array}
$$

where the Euler-Lagrange operators with respect to derivatives of $u^{\alpha}$ are obtained from (5) by replacing $u^{\alpha}$ by the corresponding derivatives. For example,

$$
\begin{array}{r}
\frac{\delta}{\delta u_{i}^{\alpha}}=\frac{\partial}{\partial u_{i}^{\alpha}}+\sum_{s \geq 1}(-1)^{s} D_{j_{1}} \cdots D_{j_{s}} \frac{\partial}{\partial u_{i j_{1} j_{2} \cdots j_{s}}^{\alpha}}, \\
i=1, \ldots, n, \quad \alpha=1, \ldots, m,
\end{array}
$$

and the Euler-Lagrange, Lie-Bäcklund, and Noether operators are connected by the operator identity:

$$
X+D_{i}\left(\xi^{i}\right)=W^{\alpha} \frac{\delta}{\delta u^{\alpha}}+D_{i} N^{i}
$$

The $n$-tuple vector $T=\left(T^{1}, T^{2}, \ldots, T^{n}\right), T^{j} \in \mathscr{A}, j=1, \ldots, n$ is a conserved vector of (3) if $T^{i}$ satisfies

$$
\left.D_{i} T^{i}\right|_{(3)}=0 \text {. }
$$

Equation (14) defines a local conservation law of system (3).

2.2. Lie Point Symmetries. Now, we consider the following Lie group of transformations with independent variables $x, y, t$ and dependent variable $u$ :

$$
\begin{array}{rlrl}
x^{*} & =x^{*}(x, y, t, \varepsilon), & y^{*}=y^{*}(x, y, t, \varepsilon), \\
t^{*}=t^{*}(x, y, t, \varepsilon), & u^{*}=u^{*}(x, y, t, \varepsilon),
\end{array}
$$

where $\varepsilon$ is the group parameter. The infinitesimal generator for the Lie group transformations can be expressed in the following form:

$$
X=\xi \frac{\partial}{\partial x}+\phi \frac{\partial}{\partial y}+\tau \frac{\partial}{\partial t}+\eta \frac{\partial}{\partial u} .
$$

Applying the fourth prolongation $\mathrm{pr}^{(4)} X$ to (1), we obtain the following determining equations:

$$
\begin{gathered}
\xi_{y}=\xi_{t}=\xi_{u}=\phi_{x}=\phi_{u}=\tau_{x}=\tau_{u}=0, \\
\xi_{x x}=\eta_{x x}=\eta_{x u}=\eta_{u u}=0, \\
\tau_{t}-\phi_{y}=0, \quad \tau_{t}-2 \xi_{x}=0, \quad \phi_{t}-\tau_{y}=0, \\
\tau_{t t}+2 \eta_{u t}+\tau_{y y}=0, \quad \phi_{t t}-2 \eta_{u t}+\phi_{y y}=0, \\
\eta_{x x}+\eta_{y y}-\eta_{t t}+\eta_{x x x x}=0, \\
\eta-\xi_{x}+\tau_{t}+2 u\left(\tau_{t}-\xi_{x}\right)=0,
\end{gathered}
$$$$
\eta_{u}+2 \tau_{t}-2 \xi_{x}=0, \quad 4 \eta_{x}+\xi_{t t}+2 \eta_{x u}-\xi_{y y}=0 .
$$ 
Solving the above system (17) we reach that the symmetry of (1) is spanned by the five vector fields:

$$
\begin{gathered}
X_{1}=\frac{\partial}{\partial x}, \quad X_{2}=\frac{\partial}{\partial y}, \quad X_{3}=\frac{\partial}{\partial t}, \\
X_{4}=y \frac{\partial}{\partial t}+t \frac{\partial}{\partial y}, \\
X_{5}=x \frac{\partial}{\partial x}+2 y \frac{\partial}{\partial y}+2 t \frac{\partial}{\partial t}+(2 u+1) \frac{\partial}{\partial u} .
\end{gathered}
$$

2.3. Group-Invariant Solutions. In this section we present the reduction forms of (1) by using symmetry group method. To do this, particular linear combinations of infinitesimals are considered and their corresponding invariants are determined. For example, for the symmetry operator $X=X_{1}+$ $X_{2}+X_{3}$, we can compute the invariants by integrating the characteristic equations:

$$
\frac{d x}{1}=\frac{d y}{1}=\frac{d t}{1} .
$$

The corresponding invariants are $q=x-y, p=t-y, \omega=$ $u$. Now treating $p, q$ as the new independent variables and $\omega$ as the new dependent variable, we obtain the ordinary differential equation:

$$
(2 \omega(q)+1) \omega^{\prime \prime}(q)+2\left(\omega^{\prime}(q)\right)^{2}+\omega^{(4)}(q)=0 .
$$

Integrating (20) twice with respect to $q$ we obtain

$$
\omega^{\prime \prime}(q)+\omega^{2}(q)+\omega(q)=c_{0} q+c_{1}, \quad c_{0}, c_{1} \in R .
$$

Taking $c_{0}=0$ and $\omega^{\prime}(q)=\psi(\omega)$ we get

$$
\psi^{\prime}(\omega) \psi(\omega)+\omega^{2}+\omega=c_{1} .
$$

The solution of (22) is

$$
\psi(\omega)= \pm \frac{1}{3} \sqrt{-6 \omega^{3}+18 c_{1} \omega-9 \omega^{2}+9 c_{2}},
$$

where $c_{2}$ is a constant. Notice that $\omega^{\prime}(q)=\psi(\omega)$; we have

$$
\omega^{\prime}(q)= \pm \frac{1}{3} \sqrt{-6 \omega^{3}+18 c_{1} \omega-9 \omega^{2}+9 c_{2}},
$$

so we get

$$
q= \pm 3 \int \frac{1}{\sqrt{-6 \omega^{3}+18 c_{1} \omega-9 \omega^{2}+9 c_{2}}} d \omega .
$$

Recall $q=x-y, \omega=u$; we get the solution of (1):

$$
x-y= \pm 3 \int \frac{1}{\sqrt{-6 u^{3}+18 c_{1} u-9 u^{2}+9 c_{2}}} d u .
$$

\section{Conservation Law}

Here we briefly present the pertinent results of the two variational methods we utilize below.
3.1. Variational Method for a System and Its Adjoint. The system of adjoint equations to the system of $k$ th-order differential equation (3) is defined by [23]

$$
E_{\alpha}^{*}\left(x, u, v, \ldots, u_{(k)}, v_{(k)}\right)=0, \quad \alpha=1, \ldots, m,
$$

where

$$
\begin{array}{r}
E_{\alpha}^{*}\left(x, u, v, \ldots, u_{(k)}, v_{(k)}\right)=\frac{\delta\left(v^{\beta} E_{\beta}\right)}{\delta u^{\alpha}}, \\
\alpha=1, \ldots, m, \quad v=v(x),
\end{array}
$$

and $v=\left(v^{1}, v^{2}, \ldots, v^{m}\right)$ are new dependent variables. [23].

We recall here the following results as given in Ibragimov

A system of (3) is said to be self-adjoint if the substitution of $v=u$ into the system of adjoint equation (27) yields the same system (3).

Assume the system of (3) admits the symmetry generator:

$$
X=\xi^{i} \frac{\partial}{\partial x^{i}}+\eta^{\alpha} \frac{\partial}{\partial u^{\alpha}},
$$

and then the system of adjoint equation (27) admits the operator:

$$
\begin{gathered}
Y=\xi^{i} \frac{\partial}{\partial x^{i}}+\eta^{\alpha} \frac{\partial}{\partial u^{\alpha}}+\eta_{*}^{\alpha} \frac{\partial}{\partial \nu^{\alpha}}, \\
\eta_{*}^{\alpha}=-\left[\lambda_{\beta}^{\alpha} \nu^{\beta}+\nu^{\alpha} D_{i}\left(\xi^{i}\right)\right],
\end{gathered}
$$

where the operator (30) is an extension of (29) to the variable $\nu^{\alpha}$ and the $\lambda_{\beta}^{\alpha}$ are obtainable from

$$
X\left(E_{\alpha}\right)=\lambda_{\alpha}^{\beta} E_{\beta} .
$$

Theorem 1 (see [23]). Every Lie point, Lie-Bäcklund, and nonlocal symmetry (29) admitted by the system of (3) gives rise to a conservation law for the system consisting of (3) and the adjoint equation (27), where the components $T^{i}$ of the conserved vector $T=\left(T^{1}, \ldots, T^{n}\right)$ are determined by

$$
\begin{array}{r}
T^{i}=\xi^{i} L+W^{\alpha} \frac{\delta L}{\delta u_{i}^{\alpha}}+\sum_{s \geq 1} D_{i_{1}} \cdots D_{i_{s}}\left(W^{\alpha}\right) \frac{\delta L}{\delta u_{i i_{1} i_{2} \cdots i_{s}}^{\alpha}}, \\
i=1, \ldots, n,
\end{array}
$$

with Lagrangian given by

$$
L=\nu^{\alpha} E_{\alpha}\left(x, u, \ldots, u_{(k)}\right) .
$$

3.2. Partial Noether Approach for a System of PDEs. The following results are due to Kara and Mahomed [26] that are based on the partial Lagrangian approach to construct conservation laws for a system of PDEs.

Suppose that the system of (3) is written as

$$
E_{\alpha}=E_{\alpha}^{0}+E_{\alpha}^{1}=0, \quad \alpha=1, \ldots, m .
$$


If there exist a function $L=L\left(x, u, u_{(1)}, \ldots, u_{(l)}\right) \in \mathscr{A}, l \leq k$ and nonzero functions $f_{\alpha}^{\beta} \in \mathscr{A}$ such that (34) can be written as $\delta L / \delta u^{\alpha}=f_{\alpha}^{\beta} E_{\beta}^{1}$, provided $E_{\beta}^{1} \neq 0$, then $L$ is known as a partial Lagrangian of (34); otherwise it is the standard Lagrangian. The differential equations of the form

$$
\frac{\delta L}{\delta u^{\alpha}}=f_{\alpha}^{\beta} E_{\beta}^{1}
$$

are called a system of partial Euler-Lagrange equations.

The operator $X$ in (10) is a partial Noether operator corresponding to a partial Lagrangian $L \in \mathscr{A}$ of the system (35) if it can be determined from

$$
X(L)+L D_{i}\left(\xi^{i}\right)=W^{\alpha} \frac{\delta L}{\delta u^{\alpha}}+D_{i}\left(B^{i}\right),
$$

for some vector $B=\left(B^{1}, B^{2}, \ldots, B^{n}\right), B^{i} \in \mathscr{A}$. Here $W=$ $\left(W^{1}, W^{2}, \ldots, W^{m}\right), W^{\alpha} \in \mathscr{A}$ is the characteristic of $X$.

Theorem 2 (see [26]). If the operator $X$ as in (10) is a partial Noether operator of a partial Lagrangian L corresponding to a partial Eule-Lagrange system of the form (35), the components $T^{i}$ of the conserved vector $T$ of (3) or (35) can be constructed by the following formula:

$$
\begin{array}{r}
T^{i}=B^{i}-\xi^{i} L-W^{\alpha} \frac{\delta L}{\delta u_{i}^{\alpha}}-\sum_{s \geq 1} D_{i_{1}} \cdots D_{i_{s}}\left(W^{\alpha}\right) \frac{\delta L}{\delta u_{i i_{1} i_{2} \cdots i_{s}}^{\alpha}}, \\
i=1, \ldots, n,
\end{array}
$$

where the characteristic $W=\left(W^{1}, W^{2}, \ldots, W^{m}\right), W^{\alpha} \in \mathscr{A}$ of $X$ is also the characteristic of the conservation law $D_{i} T^{i}=0$ of (3).

3.3. Conservation Law. We now construct conservation law of (1) using the two approaches outlined above.

Application of the New Conservation Theorem. The $(2+1)$ dimensional Boussinesq equation is given by

$$
E_{\alpha}=u_{t t}-(2 u+1) u_{x x}-2 u_{x}^{2}-u_{y y}-u_{x x x x}=0 .
$$

We recall that (38) admits the following five Lie point symmetry generators:

$$
\begin{gathered}
X_{1}=\frac{\partial}{\partial x}, \quad X_{2}=\frac{\partial}{\partial y}, \\
X_{3}=\frac{\partial}{\partial t}, \quad X_{4}=y \frac{\partial}{\partial t}+t \frac{\partial}{\partial y}, \\
X_{5}=x \frac{\partial}{\partial x}+2 y \frac{\partial}{\partial y}+2 t \frac{\partial}{\partial t}+(2 u+1) \frac{\partial}{\partial u} .
\end{gathered}
$$

The adjoint equation of (38), by invoking (27), is

$$
E_{\alpha}^{*}=\frac{\delta\left[v\left(u_{t t}-(2 u+1) u_{x x}-2 u_{x}^{2}-u_{y y}-u_{x x x x}\right)\right]}{\delta u}=0,
$$

where $v=v(x, y, t)$ is a new dependent variable and (40) gives

$$
v_{t t}-2 u_{x x} v-4 u_{x} v_{x}-(2 u+1) v_{x x}-v_{y y}-v_{x x x x}=0 .
$$

By using Theorem 1, we obtain the following Lagrangian for the system of (38) and (40):

$$
L=v\left(u_{t t}-(2 u+1) u_{x x}-2 u_{x}^{2}-u_{y y}-u_{x x x x}\right) .
$$

(1) We first consider the Lie point symmetry generator $X_{1}=\partial / \partial x$, and we have $W=-u_{x}$. Hence using (32), we obtain the following components of the conserved vector $T$ :

$$
\begin{aligned}
& T_{x}^{1}=(2 u+2 v-1) u_{x} v_{x}-u_{x x} v_{x x}-u_{x} v_{x x x}, \\
& T_{t}^{1}=-u_{x t} v+u_{x} v_{t}, \\
& T_{y}^{1}=u_{x y} v-u_{x} v_{y} .
\end{aligned}
$$

(2) The Lie point symmetry generator $X_{2}=\partial / \partial y$ has the Lie characteristic function $W=-u_{y}$. Thus by using (32), the components of the conserved vector are given by

$$
\begin{gathered}
T_{x}^{2}=\left[2 u_{x} u_{y}+(2 u+1) u_{x y}+u_{y x x x}\right] v \\
-\left(2 u u_{y}+u_{y}\right) v_{x}-u_{x y} v_{x x}-u_{y} v_{x x x}, \\
T_{t}^{2}=-u_{y t} v+u_{y} v_{t}, \\
T_{y}^{2}=\left[u_{t t}-(2 u+1) u_{x x}-2 u_{x}^{2}\right] v-u_{y} v_{y}-v v_{x x x x} .
\end{gathered}
$$

(3) Now the Lie point symmetry generator $X_{3}=\partial / \partial t$ has the Lie characteristic function $W=-u_{t}$. Hence we can obtain the conserved vector whose components are

$$
\begin{aligned}
T_{x}^{3}= & {\left[2 u_{x} u_{t}+(2 u+1) u_{x t}+u_{x x x t}\right] v-(2 u+1) u_{t} v_{x} } \\
& -u_{x t} v_{x x}-u_{t} v_{x x x},
\end{aligned}
$$

$T_{t}^{3}=-\left[2 u_{x}^{2}+(2 u+1) u_{x x}+u_{y y}\right] v+u_{t} v_{t}-v v_{x x x x}$,

$T_{y}^{3}=-u_{y t} v-u_{t} v_{y}$.

(4) The Lie point symmetry generator $X_{4}=y(\partial / \partial t)+$ $t(\partial / \partial y)$ has the Lie characteristic function $W=$ $-t u_{y}-y u_{t}$. Hence using (32), the components of the conserved vector are given by

$$
\begin{aligned}
T_{x}^{4}=[ & 2 u_{x}\left(t u_{y}+y u_{t}\right)+(2 u+1)\left(t u_{x y}+y u_{x t}\right) \\
& \left.+t u_{x x x y}+y u_{x x x t}\right] v-(2 u+1)\left(t u_{y}+y u_{t}\right) v_{x} \\
- & \left(t u_{x y}+y u_{x t}\right) v_{x x}-\left(t u_{y}+y u_{t}\right) v_{x x x}
\end{aligned}
$$




$$
\begin{aligned}
& T_{t}^{4}=- {\left[u_{y}+2 y u_{x}^{2}+(2 u+1) y u_{x x}+y u_{y y}\right.} \\
&\left.+t u_{y t}+y u_{x x x x}\right] v+\left(t u_{y}+y u_{t}\right) v_{t}, \\
& T_{y}^{4}=\left[u_{t}-2 t u_{x}^{2}+t u_{t t}-(2 u+1) t u_{x x}+y u_{y t}-t u_{x x x x}\right] v \\
&-\left(t u_{y}+y u_{t}\right) v_{y} .
\end{aligned}
$$

(5) Finally, we consider the Lie point symmetry generator $X_{5}=x(\partial / \partial x)+2 y(\partial / \partial y)+2 t(\partial / \partial t)+(2 u+1)(\partial / \partial u)$ and the Lie characteristic function $W=(2 u+1)-$ $x u_{x}-2 y u_{y}-2 t u_{t}$. Hence we can obtain the conserved vector whose components are

$$
\begin{aligned}
T_{x}^{5}= & {\left[x u_{t t}-u_{y y}+2 u_{x}\left(2 y u_{y}+2 t u_{t}-2 u-1\right)\right.} \\
& +(4 u+2)\left(y u_{x y}+t u_{x t}\right)-u_{x x x} \\
& \left.-2 y u_{x x x y}-2 t u_{x x x t}\right] v \\
+ & (2 u+1)\left[(2 u+1)-x u_{x}-2 y u_{y}-2 t u_{t}\right] v_{x} \\
& -\left(x u_{x x}+2 y u_{x y}+2 t u_{x t}\right) v_{x x} \\
+ & {\left[(2 u+1)-x u_{x}-2 y u_{y}-2 t u_{t}\right] v_{x x x}, } \\
T_{t}^{5}=v & {\left[-2(2 u+1) t u_{x x}-4 t u_{x}^{2}-2 t u_{y} y\right.} \\
& \left.-2 t u_{x x x x}-x u_{x t}-2 y u_{y t}\right] \\
& -v_{t}\left[(2 u+1)-x u_{x}-2 y u_{y}-2 t u_{t}\right], \\
T_{y}^{5}=v & {\left[2 y u_{t t}-2 y(2 u+1) u_{x x}-4 y u_{x}^{2}\right.} \\
& \left.-2 y u_{x x x x}+x u_{x y}+2 t u_{y t}\right] \\
+ & v_{y}\left(2 u+1-x u_{x}-2 y u_{y}-2 t u_{t}\right) .
\end{aligned}
$$

Remark 3. The conserved vector contains the arbitrary solution $v$ of the adjoint equation (40) and hence one can obtain an infinite number of conservation laws.

Application of the Partial Lagrange Method. Consider the partial Lagrange approach given by Kara and Mahomed [26]. A partial Lagrange for (1) is

$$
L=\frac{1}{2} u_{t}^{2}-\left(u+\frac{1}{2}\right) u_{x}^{2}-\frac{1}{2} u_{y}^{2}-\frac{1}{2} u_{x x}^{2}
$$

The Euler-Lagrange-type equation is

$$
\frac{\delta L}{\delta u}=-u_{t t}+(2 u+1) u_{x x}+u_{x}^{2}+u_{y y}+u_{x x x x} .
$$

So (1) can be written as

$$
\frac{\delta L}{\delta u}=-u_{x}^{2}
$$

to determine the Noether-type operators. If we substitute (48) and (50) into the partial Noether-type operators determining (36), we obtain

$$
\begin{aligned}
\xi & =\phi=\tau=\eta_{u}=0, \quad \eta=C(x, y, t), \\
B^{1} & =-C_{t} u+\alpha(x, y, t), \\
B^{2} & =-\left(u^{2}+u\right) C_{x}+\beta(x, y, t), \\
B^{3} & =-C_{y} u+\gamma(x, y, t),
\end{aligned}
$$

subject to the condition

$$
B_{t}^{1}+B_{x}^{2}+B_{y}^{3}=0 .
$$

The conserved components, using (37), are

$$
\begin{aligned}
& T^{1}=C_{t} u-C u_{t}+\alpha, \\
& T^{2}=-\left(u^{2}+u\right) C_{x}+C(2 u+1) u_{x}+C_{x} u_{x x}+\beta, \\
& T^{3}=-C_{y} u+C u_{y}+\gamma .
\end{aligned}
$$

\section{Conclusions}

In this paper, we investigate Lie point symmetries, similarity reduction, invariant solutions, and conservation laws of the $(2+1)$-dimensional Boussinesq equation. One of the most important applications of theory of Lie groups is to obtain the conservation laws of differential equations. It is well known that the famous Noether theorem establishes a connection between symmetries and conservation laws of differential equations provided that the equations are Euler-Lagrange equations. However, (1) does not admit Lagrangians. We obtain conservation laws of (1) by utilizing the new conservation theorem and the partial Lagrange approach.

\section{Conflict of Interests}

The authors declare that there is no conflict of interests regarding the publication of this paper.

\section{Acknowledgments}

This work was supported by the Fundamental Research Funds for the Central Universities (no. 2013XK03) and the National Natural Science Foundation of China (Grant no. 11371361).

\section{References}

[1] M. J. Ablowitz and P. A. Clarkson, Solitons, Nonlinear Evolution Equations and Inverse Scattering, Cambridge University Press, New York, NY, USA, 1991.

[2] V. B. Matveev and M. A. Salle, Darboux Transformations and Solitons, Springer, Berlin, Germany, 1991.

[3] Y. Chen, Z. Y. Yan, and H. Q. Zhang, "Exact solutions for a family of variable-coefficient reaction-duffing equations via the Backlund transformation," Theoretical and Mathematical Physics, vol. 132, no. 1, pp. 970-975, 2002. 
[4] A. Wazwaz, "The Hirota's direct method for multiple-soliton solutions for three model equations of shallow water waves," Applied Mathematics and Computation, vol. 201, no. 1-2, pp. 489-503, 2008.

[5] E. Fan and H. Zhang, "A note on the homogeneous balance method," Physics Letters A, vol. 246, no. 5, pp. 403-406, 1998.

[6] E. Fan, "Two new applications of the homogeneous balance method," Physics Letters A, vol. 265, no. 5-6, pp. 353-357, 2000.

[7] S. Y. Lou, H. Y. Ruan, D. F. Chen, and W. Z. Chen, "Similarity reductions of the KP equation by a direct method," Journal of Physics A: Mathematical and General, vol. 24, no. 7, pp. 14551467, 1991.

[8] S. Lou, X. Tang, and J. Lin, "Similarity and conditional similarity reductions of a $(2+1)$-dimensional $\mathrm{KdV}$ equation via a direct method," Journal of Mathematical Physics, vol. 41, no. 12, pp. 8286-8303, 2000.

[9] A. Wazwaz, "The tanh method for traveling wave solutions of nonlinear equations," Applied Mathematics and Computation, vol. 154, no. 3, pp. 713-723, 2004.

[10] E. Yusufoglu and A. Bekir, "Solitons and periodic solutions of coupled nonlinear evolution equations by using the sine-cosine method," International Journal of Computer Mathematics, vol. 83, no. 12, pp. 915-924, 2006.

[11] P. E. Hydon, Symmetry Methods for Differential Equations: A Beginners Guide, Cambridge University Press, Cambridge, UK, 2000.

[12] F. M. Mahomed, "Symmetry group classification of ordinary differential equations: survey of some results," Mathematical Methods in the Applied Sciences, vol. 30, no. 16, pp. 1995-2012, 2007.

[13] R. Hirota, "Direct methods in soliton theory," in Solitons, R. K. Bullogh and P. J. Caudrey, Eds., Springer, Berlin, Germany, 1980.

[14] R. Hirota, "Exact envelope-soliton solutions of a nonlinear wave equation," Journal of Mathematical Physics, vol. 14, pp. 805-809, 1973.

[15] E. V. Krishnan, S. Kumar, and A. Biswas, "Solitons and other nonlinear waves of the Boussinesq equation," Nonlinear Dynamics, vol. 70, no. 2, pp. 1213-1221, 2012.

[16] H. W. Yang, B. S. Yin, and Y. L. Shi, "Forced dissipative Boussinesq equation for solitary waves excited by unstable topography," Nonlinear Dynamics, vol. 70, no. 2, pp. 1389-1396, 2012.

[17] S. M. El-Sayed and D. Kaya, "The decomposition method for solving (2+1)-dimensional Boussinesq equation and (3+1)dimensional KP equation," Applied Mathematics and Computation, vol. 157, no. 2, pp. 523-534, 2004.

[18] M. Senthilvelan, "On the extended applications of homogeneous balance method," Applied Mathematics and Computation, vol. 123, no. 3, pp. 381-388, 2001.

[19] Y. Chen, Z. Yan, and H. Zhang, "New explicit solitary wave solutions for $(2+1)$-dimensional Boussinesq equation and $(3+$ 1)-dimensional KP equation," Physics Letters A, vol. 307, no. 2-3, pp. 107-113, 2003.

[20] C. Huai-Tang and Z. Hong-Qing, "New double periodic and multiple soliton solutions of the generalized $(2+1)$-dimensional Boussinesq equation," Chaos, Solitons and Fractals, vol. 20, no. 4, pp. 765-769, 2004.

[21] G. W. Bluman and S. Kumei, Symmetries and Differential Equations, vol. 81 of Applied Mathematical Sciences, Springer, New York, NY, USA, 1989.
[22] P. J. Olver, Applications of Lie Groups to Differential Equations, vol. 107 of Graduate Texts in Mathematics, Springer, Berlin, Germany, 2nd edition, 1993.

[23] N. H. Ibragimov, "A new conservation theorem," Journal of Mathematical Analysis and Applications, vol. 333, no. 1, pp. 311328, 2007.

[24] N. H. Ibragimov and T. Kolsrud, "Lagrangian approach to evolution equations: symmetries and conservation laws," Nonlinear Dynamics, vol. 36, no. 1, pp. 29-40, 2004.

[25] A. H. Kara and F. M. Mahomed, "Relationship between symmetries and conservation laws," International Journal of Theoretical Physics, vol. 39, no. 1, pp. 23-40, 2000.

[26] A. H. Kara and F. M. Mahomed, "Noether-type symmetries and conservation laws via partial Lagrangians," Nonlinear Dynamics, vol. 45, no. 3-4, pp. 367-383, 2006. 


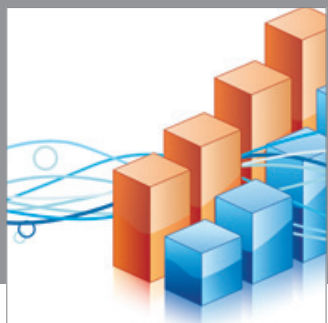

Advances in

Operations Research

mansans

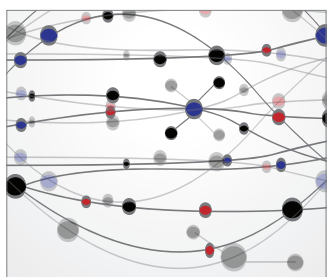

The Scientific World Journal
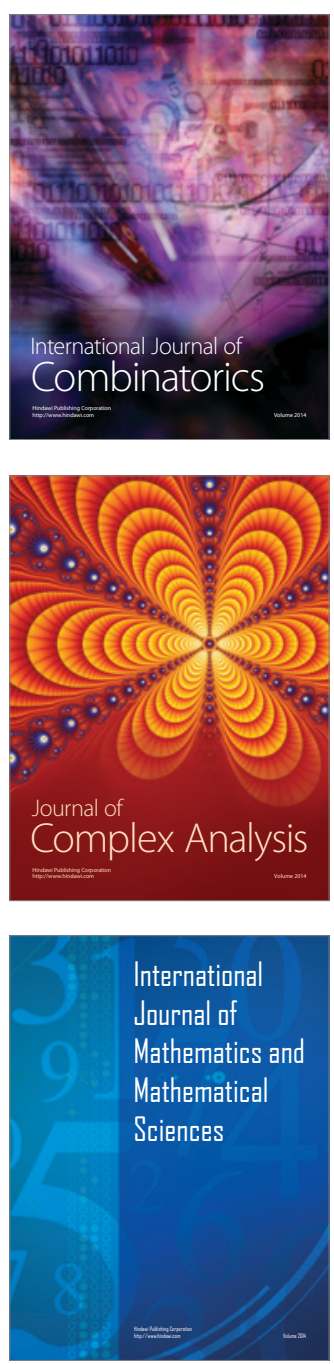
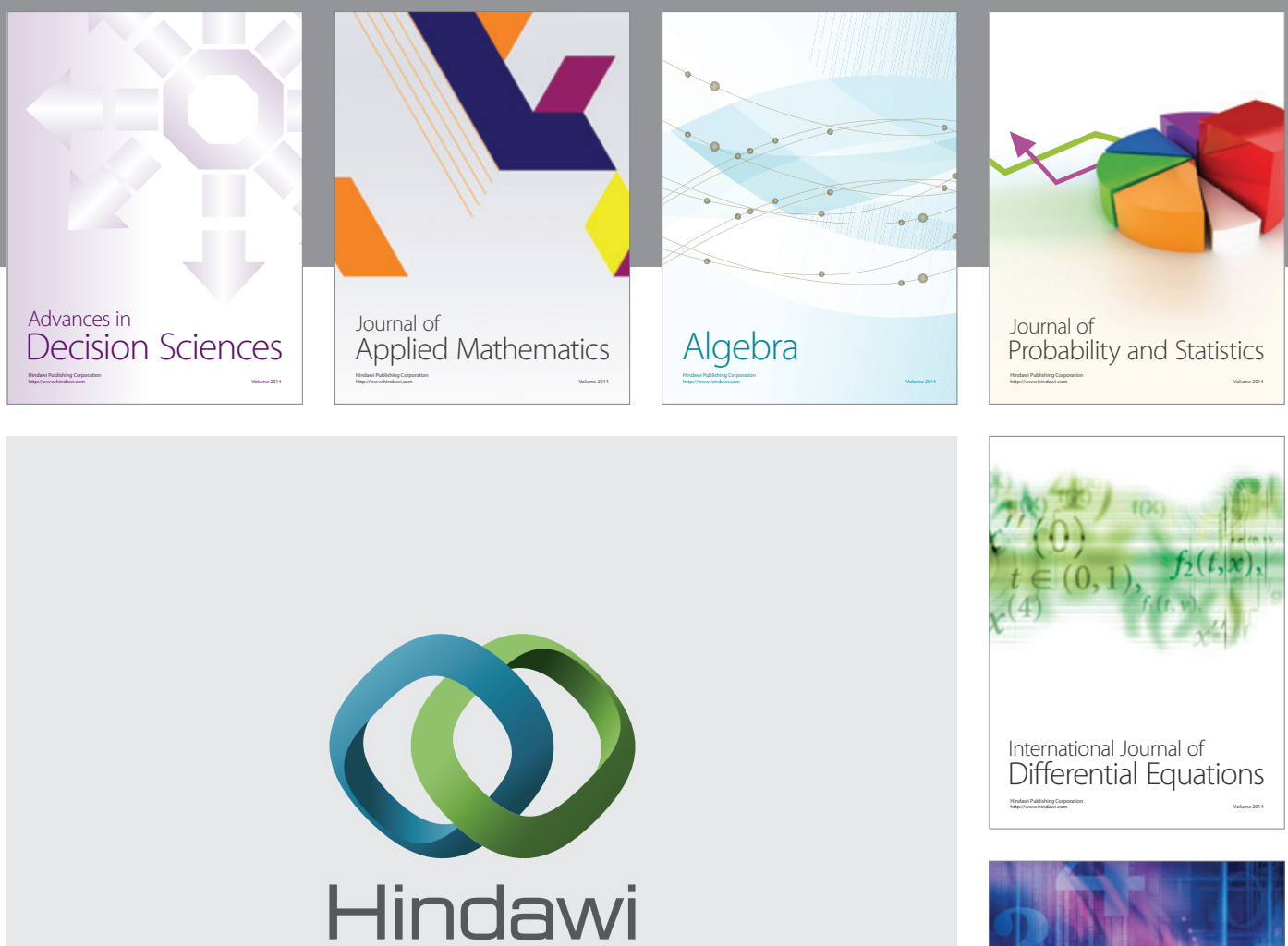

Submit your manuscripts at http://www.hindawi.com
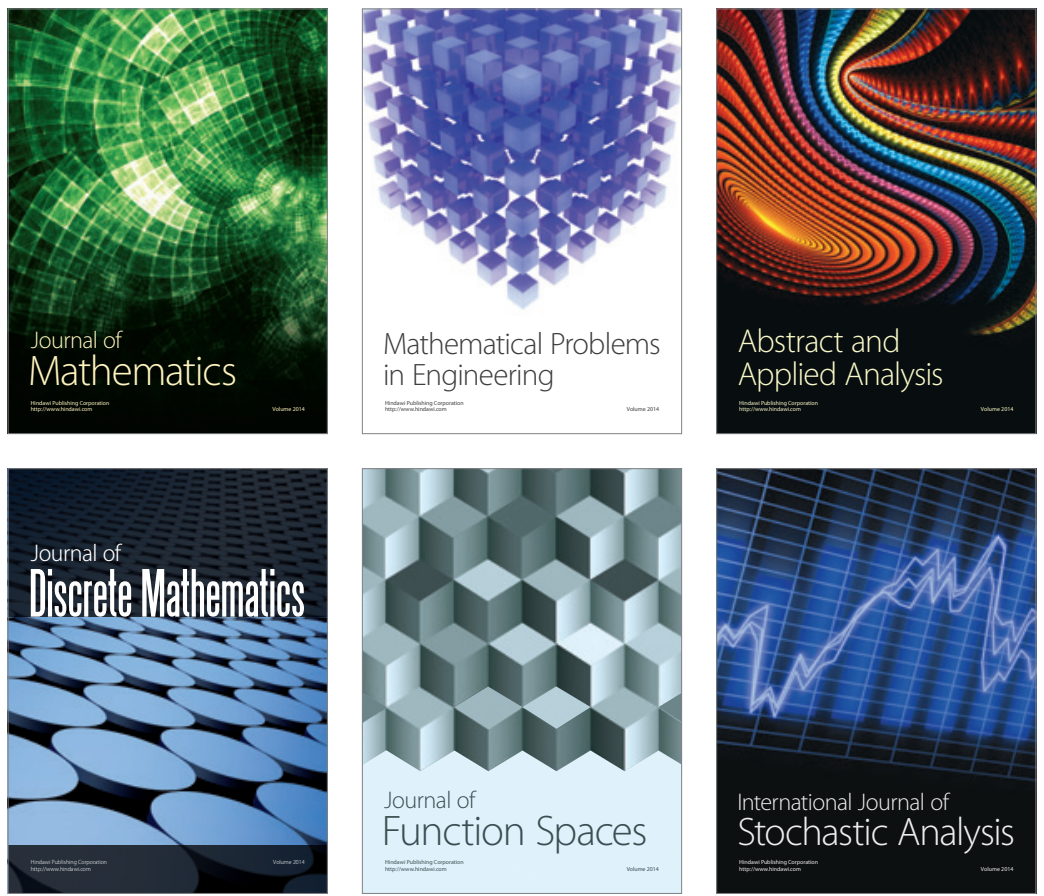

Journal of

Function Spaces



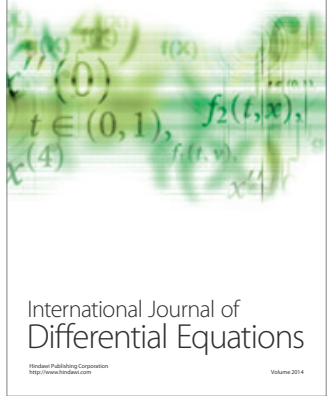
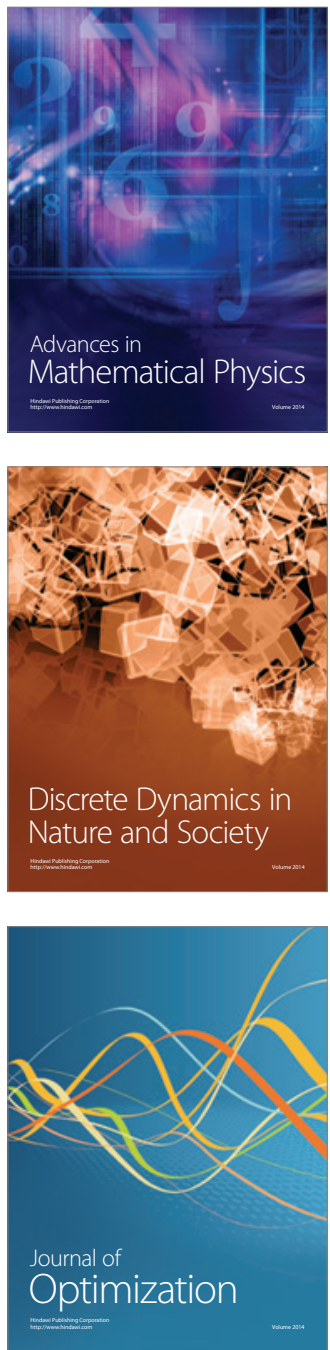